\title{
Feynman Perturbation Series for the Morse Potential
}

\author{
Boudjedaa Badredine1,2, Meftah Mohamed Tayeb3 ${ }^{3}$ Chetouani Lyazid4 \\ ${ }^{1}$ Département de Mathématiques, Faculté des Sciences exactes et Informatique, Université Med Saddik Ben \\ Yahia, Jijel, Algérie \\ ${ }^{2}$ Laboratoire de Mathématiques Appliquées, Université Kasdi Merbah, Ouargla, Algérie \\ ${ }^{3}$ Département de Physique, Faculté des Sciences et des Sciences de I'Ingénieur, Laboratoire LRPPS Université \\ Kasdi Merbah, Ouargla, Algérie \\ ${ }^{4}$ Département de Physique, Université de Constantine I, Constantine, Algérie \\ Email: badredineb@gmail.com, mewalid@yahoo.com, lyazidchetouani@gmail.com
}

Received 14 December 2013; revised 12 January 2014; accepted 9 February 2014

Copyright (C) 2014 by authors and Scientific Research Publishing Inc.

This work is licensed under the Creative Commons Attribution International License (CC BY).

http://creativecommons.org/licenses/by/4.0/

(c) (i) Open Access

\begin{abstract}
In this paper we give an alternative treatment of the Schrödinger equation with the Morse potential, which based on the exact summation of the Feynman perturbation series in its original form. Using Fourier transform we establish a recurrence equation between terms of the perturbation series. Finally, by the inverse Fourier transform and some technical tools of the ordinary differential equations of the second order, we can compute the exact sum of the perturbation series which is the Green's function of the problem.
\end{abstract}

\section{Keywords}

Morse Potential; Green's Function; Propagator; Path Integral; Perturbation Series; Fourier Transform

\section{Introduction}

In quantum mechanics, the class of potentials for which Schrödinger equation can be exactly solved has been extended considerably by using different methods. The popular and widely one used in quantum mechanics is the perturbation theory leading to solve the problems approximately. Furthermore, among problems that can be exactly solved, there are few whose solutions can be obtained exactly by summing up the perturbation series in the path integral formalism [1]. Exact Green's functions: for delta-function [2]-[4], for Coulomb potential [5]-[7], for the inverse square potential [8] and for the step potential [9] are obtained by summing up the pertur- 
bation series in the path integral framework. In [2], the Feynman perturbation series are used to study the one-dimensional delta-function potential, where the authors extracted only correct informations for wave functions but they did not give the exact expression form of the propagator. The use of the same technique, perturbation series, gave the exact expression for the propagator for the delta-function potential [3]. We can find several examples of potentials problem with a delta-function perturbation by means of path integrals [4], the Green's function for each problem is derived by summing the Feynman perturbation series. In [5], the perturbation series are used to derive the Green's function for the Coulomb potential in a closed analytical form. The Green's function of the one-dimensional relativistic Wood-Saxon, step and square well potential are evaluate by the Kleinert's path integral technique [6] and in [7] the same author has calculated the Green's function of the D-dimensional Coulomb by summing exactly the perturbation series; the energy spectra and wave functions are extracted. The exact propagator is derived by summing the Feynman perturbation series for a particle moving in the inverse square potential [8]. The Green's function for the step potential is given by the exact summation of the perburbation series [9].

The Morse potential is one of the important potentials in physics, which raises many interests in many areas specialy in molecular physics and is used for the description of the interaction between the atoms in diatomic molecules. The Schrödinger equation for the Morse potential has been solved exactly or studied by different methods recently, for example, [10]-[18].

In the paper [19], we have derived the Green's function of the Morse one-dimensional potential using the perturbation series, not by summing exactly the series but we use its termes to the final result. The news is that we have presented the use of the Fourier transform in the Feymann path integral perturbation series method.

In this work, we will use the same technique in [19] and some results of the ordinary differential equations of the second ordre. We calculate the Green's function of the problem by computing the exact sum of the perturbation series, but in a different way as in [19].

\section{Path Integral for the Morse Potential via the Sum of the Perturbation Series}

We are interested to calculate the propagator, say the Green's function relative to the one-dimensional Morse potential:

$$
V(x)=V_{0}(\exp (-2 x)-2 \exp (-x))
$$

which can be written as:

$$
V(x)=4 V_{0} \sum_{s=1}^{2}\left(-\frac{1}{2}\right)^{s} \exp (-s x)
$$

where $V_{0}>0$ is the strength of the potential. The Feynman propagator is defined, taking $\hbar=1$, by:

$$
K\left(x, T / x_{0}, 0\right)=\int_{x(0)=0}^{x(T)=x} D[x(t)] \exp \left(i \int_{0}^{T} L(x, \dot{x}, t) \mathrm{d} t\right)
$$

where $L$ is the Lagrangian of the problem and $D[x(t)]$ is the formal measure on the path space. If we split the Lagrangian into the free part and the interaction part as (in unit mass):

$$
L(x, \dot{x}, t)=\frac{\dot{x}^{2}}{2}-V(x)
$$

We can show that the Feynman propagator takes the form:

$$
K\left(x, T / x_{0}, 0\right)=\sum_{n=0}^{\infty}(i)^{n} K_{n}\left(x, T / x_{0}, 0\right)
$$

where:

$$
K_{n}\left(x, T / x_{0}, 0\right)=(-1)^{n} \int_{0}^{T} \mathrm{~d} t_{n} \cdots \int_{0}^{t_{2}} \mathrm{~d} t_{1} \int_{-\infty}^{+\infty} \cdots \int_{-\infty}^{+\infty} \prod_{j=0}^{n} K_{0}\left(x_{j+1}, t_{j+1} / x_{j}, t_{j}\right) \prod_{j=1}^{n} V\left(x_{j}\right) \mathrm{d} x_{j}
$$

And $K_{0}\left(x_{j+1}, t_{j+1} / x_{j}, t_{j}\right)$ is the free particle propagator given by: 


$$
K_{0}\left(x_{j+1}, t_{j+1} / x_{j}, t_{j}\right)=\left(\frac{1}{2 i \pi\left(t_{j+1}-t_{j}\right)}\right)^{1 / 2} \exp \left(\frac{i\left(x_{j+1}-x_{j}\right)^{2}}{2\left(t_{j+1}-t_{j}\right)}\right)
$$

Taking the Fourier transform of $K_{n}\left(x, T / x_{0}, 0\right)$ on $T$ as:

$$
G_{n}\left(x, x_{0} ; E\right)=G_{n}\left(x, x_{0}\right)=\frac{1}{i} \int_{0}^{+\infty} K_{n}\left(x, T / x_{0}, 0\right) \exp (i E T) \mathrm{d} T
$$

we write this last formula as:

$$
G_{n}\left(x, x_{0}\right)=i \int_{-\infty}^{+\infty} \mathrm{d} x_{n} G_{0}\left(x, x_{n}\right) V\left(x_{n}\right) G_{n-1}\left(x_{n}, x_{0}\right)
$$

where:

$$
G_{0}\left(x, x_{n}\right)=\frac{1}{i} \int_{0}^{+\infty} K_{0}\left(x, T / x_{n}, 0\right) \exp (i E T) \mathrm{d} T=\frac{1}{i} \int_{0}^{+\infty} \sqrt{\frac{1}{2 i \pi T}} \exp \left(i E T+\frac{i}{2 T}\left(x-x_{n}\right)^{2}\right) \mathrm{d} T
$$

and using Equations (1) and (9), then (8) becomes:

$$
\begin{gathered}
G_{n}\left(x, x_{0}\right)=-4 V_{0} \sum_{s=1}^{2}\left(-\frac{1}{2}\right)^{s} \int_{0}^{+\infty} \sqrt{\frac{1}{2 i \pi T}} \exp (i E T) \mathrm{d} T \int_{-\infty}^{+\infty} \mathrm{d} x_{n} \exp \left(-s x_{n}+\frac{i}{2 T}\left(x-x_{n}\right)^{2}\right) G_{n-1}\left(x_{n}, x_{0}\right) \\
G_{n}\left(x, x_{0}\right)=-4 V_{0} \sum_{s=1}^{2}\left(-\frac{1}{2}\right)^{s} \exp (-s x) \int_{0}^{+\infty} \sqrt{\frac{1}{2 i \pi T}} \exp (i E T) \mathrm{d} T \int_{-\infty}^{+\infty} \mathrm{d} x_{n} \exp \left(s\left(x-x_{n}\right)+\frac{i}{2 T}\left(x-x_{n}\right)^{2}\right) G_{n-1}\left(x_{n}, x_{0}\right)
\end{gathered}
$$

we take now the Fourier transform on the end point $x$ in the last formula, and using the convolution theorem for Fourier transform, we get:

$$
\tilde{G}_{n}\left(w, x_{0}\right)=-4 V_{0} \sum_{s=1}^{2}\left(-\frac{1}{2}\right)^{s} \int_{0}^{+\infty} \mathrm{d} T \exp \left(i T\left(E-\frac{\omega^{2}}{2}\right)\right) \tilde{G}_{n-1}\left(\omega+i s, x_{0}\right)
$$

i.e.

$$
\tilde{G}_{n}\left(w, x_{0}\right)=2 i V_{0} f_{0}(\omega)\left[\tilde{G}_{n-1}\left(\omega+2 i, x_{0}\right)-2 \tilde{G}_{n-1}\left(\omega+i, x_{0}\right)\right], n \geq 1
$$

where:

$$
\tilde{G}_{n}\left(w, x_{0}\right)=\int_{-\infty}^{+\infty} \exp (i \omega x) G_{n}\left(x, x_{0}\right) \mathrm{d} x
$$

and

$$
f_{0}(\omega)=i \int_{0}^{+\infty} \mathrm{d} T \exp i T\left(E-\frac{\omega^{2}}{2}\right)=\frac{2}{2 \varepsilon^{2}+\omega^{2}} \text {, where } E=-\varepsilon^{2}
$$

From Equation (12), we see that all termes $\tilde{G}_{n}\left(w, x_{0}\right)$ are known and depend on the expression of $\tilde{G}_{0}\left(\omega+i n, x_{0}\right)$ which is:

$$
\begin{aligned}
\tilde{G}_{0}\left(\omega+i n, x_{0}\right) & =\int_{-\infty}^{+\infty} \exp (i(\omega+i n) x) G_{0}\left(x, x_{0}\right) \mathrm{d} x=-\left(\frac{2}{2 \varepsilon^{2}+(\omega+i n)^{2}}\right) \exp (i \omega-n) x_{0} \\
& =-2 f_{n}(\omega) \exp (i \omega-n) x_{0} .
\end{aligned}
$$

where we note $f_{n}(\omega)$ by:

$$
f_{n}(\omega)=\frac{1}{2 \varepsilon^{2}+(\omega+i n)^{2}}, \quad n=1,2, \cdots
$$


Let now compute the first and the second terms of Equation (12):

$$
\tilde{G}_{1}\left(w, x_{0}\right)=2 i V_{0} f_{0}(\omega) \exp \left(i \omega x_{0}\right)\left[f_{2}(\omega) \exp \left(-2 x_{0}\right)-2 f_{1}(\omega) \exp \left(-x_{0}\right)\right]
$$

and

$$
\begin{aligned}
\tilde{G}_{2}\left(w, x_{0}\right)= & 2^{2} V_{0}^{2} f_{0}(\omega) \exp \left(i \omega x_{0}\right)\left[f_{2}(\omega) f_{4}(\omega) \exp \left(-4 x_{0}\right)-2 f_{2}(\omega) f_{3}(\omega) \exp \left(-3 x_{0}\right)\right. \\
& \left.-2 f_{1}(\omega) f_{3}(\omega) \exp \left(-3 x_{0}\right)+4 f_{1}(\omega) f_{2}(\omega) \exp \left(-2 x_{0}\right)\right]
\end{aligned}
$$

and so on, we can see that all terms $\tilde{G}_{n}\left(w, x_{0}\right)$ are determined in a linear combination of $f_{n}(\omega)$ and powers of $\exp \left(-x_{0}\right)$ Since $\tilde{G}\left(w, x_{0}\right)$ is:

$$
\tilde{G}\left(w, x_{0}\right)=\sum_{n=0}^{\infty}(i)^{n} \tilde{G}_{n}\left(w, x_{0}\right)
$$

and if we bring together all terms in power of $\exp \left(-x_{0}\right)$, we get:

$$
\tilde{G}\left(w, x_{0}\right)=f_{0}(\omega) \exp \left(i \omega x_{0}\right)\left[\sum_{n=0}^{\infty} a_{n}(\omega) \exp \left(-n x_{0}\right)\right]
$$

where the coefficients $a_{n}(\omega)$ satisfy the recurrence formula:

$$
a_{n}(\omega)=2 V_{0} f_{n}(\omega)\left[2 a_{n-1}(\omega)-a_{n-2}(\omega)\right]
$$

or:

$$
\left[2 \varepsilon^{2}+\omega^{2}-n^{2}+2 i n \omega\right] a_{n}(\omega)=2 V_{0}\left[2 a_{n-1}(\omega)-a_{n-2}(\omega)\right]
$$

with $a_{-1}=0, a_{0}=-1, a_{n}(\omega)=-4 V_{0} f_{1}(\omega), \cdots$, etc .

Now noting the series in Equation (17) by:

$$
f_{0}(\omega) F(X)=f_{0}(\omega) \sum_{n=0}^{\infty} a_{n}(\omega) X^{n} \equiv \tilde{G}\left(\omega, x_{0}\right) \exp \left(-i \omega x_{0}\right)
$$

we can easily check that $F(X)$ which is the generating function of $a_{n}(\omega)$ satisfies the differential equation:

$$
X^{2} \frac{\mathrm{d}^{2}}{\mathrm{~d} x^{2}} F(X)+X(1-2 i \omega) \frac{\mathrm{d}}{\mathrm{d} x} F(X)-\left(2 \varepsilon^{2}+\omega^{2}-4 X V_{0}+2 X^{2} V_{0}\right) F(X)=2 \varepsilon^{2}+\omega^{2}
$$

Here we have to note that this equation is equivalent to those governing Green's function itself but written in an other form where we have put $X=\exp \left(-x_{0}\right)$ and done the Fourier transform on the end point $x$, i.e.:

$$
\left[-\frac{1}{2} \frac{\mathrm{d}^{2}}{\mathrm{~d} x_{0}^{2}}+V\left(x_{0}\right)+\varepsilon^{2}\right] G\left(x, x_{0} / E\right)=-\delta\left(x_{0}-x\right)
$$

Return now to Equation (17) and if we take the inverse Fourier Transorm on $\tilde{G}\left(w, x_{0}\right)$, we obtain:

$$
G\left(x, x_{0}\right)=\frac{1}{2 \pi} \sum_{n=0}^{\infty} \int_{-\infty}^{+\infty} \mathrm{d} \omega \exp \left(-i \omega\left(x-x_{0}\right)\right) f_{0}(\omega) a_{n}(\omega) \exp \left(-n x_{0}\right)
$$

Then if we note by:

$$
A_{n}\left(x-x_{0}\right)=\frac{1}{2 \pi} \int_{-\infty}^{+\infty} \mathrm{d} \omega \exp \left(-i \omega\left(x-x_{0}\right)\right) f_{0}(\omega) a_{n}(\omega)
$$

we see that:

$$
G\left(x, x_{0}\right)=\sum_{n=0}^{\infty} A_{n}\left(x-x_{0}\right) \exp \left(-n x_{0}\right)
$$

and with the Fourier transform properties, we have: 


$$
\begin{aligned}
& \omega^{2} f_{0}(\omega) a_{n}(\omega)=-\int_{-\infty}^{+\infty} \mathrm{d} \omega \exp (i \omega x) \frac{\mathrm{d}^{2}}{\mathrm{~d} x^{2}} A_{n}(x) \\
& i \omega f_{0}(\omega) a_{n}(\omega)=-\int_{-\infty}^{+\infty} \mathrm{d} \omega \exp (i \omega x) \frac{\mathrm{d}}{\mathrm{d} x} A_{n}(x)
\end{aligned}
$$

Then from these last formulas and the recurrence formula of $a_{n}(\omega)$ (19) we conclude that $A_{n}(x)$ satisfies:

$$
-\frac{\mathrm{d}^{2}}{\mathrm{~d} x^{2}} A_{n}(x)-2 n \frac{\mathrm{d}}{\mathrm{d} x} A_{n}(x)+\left(2 \varepsilon^{2}-n^{2}\right) A_{n}(x)=4 V_{0} A_{n-1}(x)-2 V_{0} A_{n-2}(x)
$$

with $A_{-1}(x)=0$ and $A_{0}(x)=\frac{-\exp (-\varepsilon \sqrt{2}|x|)}{\varepsilon \sqrt{2}}$, which is a linear second order ordinary differential equation with real constant coefficients. Then $A_{n}(x)$ can be expressed in term of the complementary solution plus a particular solution. Indeed, the complementary solution $A_{n}^{c}(x)$ is:

$$
A_{n}^{c}(x)=C_{n}^{1} \exp (-(n+\varepsilon \sqrt{2}) x)+C_{n}^{2} \exp (-(n-\varepsilon \sqrt{2}) x)
$$

where the coefficients $C_{n}^{1}$ and $C_{n}^{2}$ are constants independent of $x$ and using the variation of parameters method we find that the particular solution has the following expression:

$$
A_{n}^{p}(x)=C_{n}^{1}(x) \exp (-(n+\varepsilon \sqrt{2}) x)+C_{n}^{2}(x) \exp (-(n-\varepsilon \sqrt{2}) x)
$$

with $C_{n}^{1}(x), C_{n}^{2}(x)$ are determined by:

$$
\begin{aligned}
\frac{\mathrm{d}}{\mathrm{d} x} C_{n}^{1} & =\frac{V_{0}}{\varepsilon \sqrt{2}} \exp (\varepsilon \sqrt{2} x)\left[2 \exp (x) B_{n-1}(x)-\exp (2 x) B_{n-2}(x)\right] \\
\frac{\mathrm{d}}{\mathrm{d} x} C_{n}^{2} & =\frac{-V_{0}}{\varepsilon \sqrt{2}} \exp (-\varepsilon \sqrt{2} x)\left[2 \exp (x) B_{n-1}(x)-\exp (2 x) B_{n-2}(x)\right]
\end{aligned}
$$

where

$$
B_{n}(x)=A_{n}(x) \exp (n x), B_{-1}(x)=0 \text { and } B_{0}(x)=\frac{-\exp (-\varepsilon \sqrt{2}|x|)}{\varepsilon \sqrt{2}} .
$$

Finally by recurrence, we can prove that:

$$
A_{n}^{p}(x)= \begin{cases}\frac{(-1)^{n+1} V_{0}^{n}}{\varepsilon \sqrt{2}} b_{n}(\varepsilon) \exp (-\varepsilon \sqrt{2} x), & x>0 \\ \frac{(-1)^{n+1} V_{0}^{n}}{\varepsilon \sqrt{2}} b_{n}(-\varepsilon) \exp (\varepsilon \sqrt{2} x), & x<0\end{cases}
$$

where the coefficients $b_{n}(\varepsilon)$ satisfy an recurrence formula as:

$$
\left[n^{2} \pm 2 n \varepsilon \sqrt{2}\right] b_{n}(\mp \varepsilon)=4 b_{n-1}(\mp \varepsilon)+\frac{2}{V_{0}} b_{n-2}(\mp \varepsilon)
$$

with $b_{-1}(\mp \varepsilon)=0, \quad b_{0}(\mp \varepsilon)=1$.

Then from Equations (29) and (33) we have:

$$
\begin{aligned}
A_{n}(x)= & A_{n}^{p}(x)+A_{n}^{c}(x)=C_{n}^{1} \exp (-(n+\varepsilon \sqrt{2}) x)+C_{n}^{2} \exp (-(n-\varepsilon \sqrt{2}) x) \\
& +\left\{\begin{array}{ll}
\frac{(-1)^{n+1} V_{0}^{n}}{\varepsilon \sqrt{2}} b_{n}(\varepsilon) \exp (-\varepsilon \sqrt{2} x), & x>0 \\
\frac{(-1)^{n+1} V_{0}^{n}}{\varepsilon \sqrt{2}} b_{n}(-\varepsilon) \exp (\varepsilon \sqrt{2} x), & x<0
\end{array}, \quad \forall n \geq 0 .\right.
\end{aligned}
$$


we have to note that $C_{0}^{1}=C_{0}^{2}=0$.

Knowing that if the following limits exist:

$$
\lim _{x \rightarrow-\infty} \sum_{n=0}^{\infty} C_{n}^{1} \exp (-n x) \neq 0 \text { and } \lim _{x \rightarrow+\infty} \sum_{n=0}^{\infty} C_{n}^{2} \exp (-n x) \neq 0
$$

then

$$
\begin{aligned}
& \lim _{x \rightarrow \infty}\left[\exp (\varepsilon \sqrt{2} x)\left|\sum_{n=0}^{\infty} C_{n}^{2} \exp (-n x)\right|\right]=+\infty \\
& \lim _{x \rightarrow-\infty}\left[\exp (-\varepsilon \sqrt{2} x)\left|\sum_{n=0}^{\infty} C_{n}^{1} \exp (-n x)\right|\right]=+\infty
\end{aligned}
$$

So in that case and from the formulas (35), (25), we are able to write the Green's function $G\left(x, x_{0}\right)$ as:

$$
G\left(x, x_{0}\right)= \begin{cases}\exp \left(-\varepsilon \sqrt{2}\left(x-x_{0}\right)\right) \sum_{n=0}^{\infty}\left(\frac{(-1)^{n+1} V_{0}^{n}}{\varepsilon \sqrt{2}} b_{n}(\varepsilon) \exp \left(-n x_{0}\right)+C_{n}^{1}\left(x_{0}\right) \exp (-n x),\right. & x>x_{0} \\ \exp \left(\varepsilon \sqrt{2}\left(x-x_{0}\right)\right) \sum_{n=0}^{\infty}\left(\frac{(-1)^{n+1} V_{0}^{n}}{\varepsilon \sqrt{2}} b_{n}(-\varepsilon) \exp \left(-n x_{0}\right)+C_{n}^{2}\left(x_{0}\right) \exp (-n x)\right), & x_{0}>x\end{cases}
$$

Knowing that the generating functions of $b_{n}(\varepsilon)$ and $b_{n}(-\varepsilon)$ respectively are:

$$
F_{1}(X)=\sum_{n=0}^{\infty} b_{n}(\varepsilon)(-X)^{n}, \quad F_{2}(X)=\sum_{n=0}^{\infty} b_{n}(-\varepsilon)(-X)^{n} ;
$$

then if we use the recurrence formula (34) it's easy to deduce that these generating functions are given by:

$$
F_{1}(X)=X^{\varepsilon \sqrt{2}-\frac{1}{2}} G\left(2 \sqrt{\frac{2}{V_{0}} X}\right), \quad F_{2}(X)=X^{-\varepsilon \sqrt{2}-\frac{1}{2}} G\left(2 \sqrt{\frac{2}{V_{0}} X}\right)
$$

where $G(y)$ is the solution of Whittaker equation:

$$
G^{\prime \prime}(y)+\left[-\frac{1}{4}+\frac{\sqrt{2 V_{0}}}{y}+\frac{\frac{1}{4}-2 \varepsilon}{y^{2}}\right] G(y)=0
$$

and they satisfy respectively the following differential equations:

$$
\begin{gathered}
X^{2} F_{1}^{\prime \prime}(X)+X(1-2 \varepsilon \sqrt{2}) F_{1}^{\prime}(X)+2 X\left(2-\frac{X}{V_{0}}\right) F_{1}(X)=0 \\
X^{2} F_{2}^{\prime \prime}(X)+X(1+2 \varepsilon \sqrt{2}) F_{2}^{\prime}(X)+2 X\left(2-\frac{X}{V_{0}}\right) F_{2}(X)=0
\end{gathered}
$$

hence we can conclude that the Green's function $G\left(x, x_{0}\right)$ takes the form:

$$
G\left(x, x_{0}\right)=\left\{\begin{array}{l}
C_{1}(x) \exp \left(\frac{x_{0}}{2}\right) W_{\sqrt{2 V_{0}}, \varepsilon \sqrt{2}}\left[2 \sqrt{2 V_{0}} \exp \left(-x_{0}\right)\right]+\exp \left(-\varepsilon \sqrt{2}\left(x-x_{0}\right)\right) \sum_{n=0}^{\infty} C_{n}^{1}\left(x_{0}\right) \exp (-n x), \quad x>x_{0} \\
C_{2}(x) \exp \left(\frac{x_{0}}{2}\right) M_{\sqrt{2 V_{0}}, \varepsilon \sqrt{2}}\left[2 \sqrt{2 V_{0}} \exp \left(-x_{0}\right)\right]+\exp \left(\varepsilon \sqrt{2}\left(x-x_{0}\right)\right) \sum_{n=0}^{\infty} C_{n}^{2}\left(x_{0}\right) \exp (-n x), \quad x_{0}>x
\end{array}\right.
$$

where $W_{\sqrt{2 V_{0}}, \varepsilon \sqrt{2}}, M_{\sqrt{2 V_{0}}, \varepsilon \sqrt{2}}$ are the Whittaker's functions. We draw attention here that in this last formula we have taken the Whittaker's function $W_{\sqrt{2 V_{0}}, \varepsilon \sqrt{2}}$ in the case $x>x_{0}$ because it's not singular at $+\infty\left(i . e x_{0} \rightarrow-\infty\right)$, and when $x_{0}>x$ we have taken $M_{\sqrt{2 V_{0}}, \varepsilon \sqrt{2}}^{\sqrt{2 V_{0}}, \varepsilon \sqrt{2}}$ which is not singular at $0\left(i . e x_{0} \rightarrow+\infty\right)$. Let us now to go back 
to (8) i.e.:

$$
G_{n}\left(x, x_{0}\right)=i \int_{-\infty}^{+\infty} \mathrm{d} x_{n} G_{0}\left(x, x_{n}\right) V\left(x_{n}\right) G_{n-1}\left(x_{n}, x_{0}\right)
$$

for which it's obvious that:

$$
G_{n}\left(x, x_{0}\right)=i \int_{-\infty}^{+\infty} \mathrm{d} x_{1} G_{n-1}\left(x, x_{1}\right) V\left(x_{1}\right) G_{0}\left(x_{1}, x_{0}\right)
$$

from this formula and with the same way as above, if we take the Fourier transform on initial point $x_{0}$ we can see that $G\left(x, x_{0}\right)$ have another form as:

$$
G\left(x, x_{0}\right)=\left\{\begin{array}{l}
C_{1}\left(x_{0}\right) \exp \left(\frac{x}{2}\right) W_{\sqrt{2 V_{0}}, \varepsilon \sqrt{2}}\left[2 \sqrt{2 V_{0}} \exp (-x)\right]+\exp \left(-\varepsilon \sqrt{2}\left(x_{0}-x\right)\right) \sum_{n=0}^{\infty} C_{n}^{1}(x) \exp \left(-n x_{0}\right), \quad x_{0}>x \\
C_{2}\left(x_{0}\right) \exp \left(\frac{x}{2}\right) M_{\sqrt{2 V_{0}}, \varepsilon \sqrt{2}}\left[2 \sqrt{2 V_{0}} \exp (-x)\right]+\exp \left(\varepsilon \sqrt{2}\left(x_{0}-x\right)\right) \sum_{n=0}^{\infty} C_{n}^{2}(x) \exp \left(-n x_{0}\right), \quad x>x_{0}
\end{array}\right.
$$

then from Equation (42) and this last formula (44) we have for $x>x_{0}$ :

$$
C_{1}(x) \exp \left(\frac{x_{0}}{2}\right) W_{\sqrt{2 V_{0}}, \varepsilon \sqrt{2}}\left[2 \sqrt{2 V_{0}} \exp \left(-x_{0}\right)\right]=\exp \left(\varepsilon \sqrt{2}\left(x_{0}-x\right)\right) \sum_{n=0}^{\infty} C_{n}^{2}(x) \exp \left(-n x_{0}\right)
$$

and

$$
C_{2}\left(x_{0}\right) \exp \left(\frac{x}{2}\right) M_{\sqrt{2 V_{0}}, \varepsilon \sqrt{2}}\left[2 \sqrt{2 V_{0}} \exp (-x)\right]=\exp \left(-\varepsilon \sqrt{2}\left(x-x_{0}\right)\right) \sum_{n=0}^{\infty} C_{n}^{1}\left(x_{0}\right) \exp (-n x)
$$

for which Equation (44) i.e. $G\left(x, x_{0}\right)$ becomes:

$$
G\left(x, x_{0}\right)=\left\{\begin{array}{l}
C_{1}(x) \exp \left(\frac{x_{0}}{2}\right) W_{\sqrt{2 V_{0}}, \varepsilon \sqrt{2}}\left[2 \sqrt{2 V_{0}} \exp \left(-x_{0}\right)\right]+C_{2}\left(x_{0}\right) \exp \left(\frac{x}{2}\right) M_{\sqrt{2 V_{0}}, \varepsilon \sqrt{2}}\left[2 \sqrt{2 V_{0}} \exp (-x)\right], \quad x>x_{0} \\
C_{1}\left(x_{0}\right) \exp \left(\frac{x}{2}\right) W_{\sqrt{2 V_{0}}, \varepsilon \sqrt{2}}\left[2 \sqrt{2 V_{0}} \exp (-x)\right]+C_{2}(x) \exp \left(\frac{x_{0}}{2}\right) M_{\sqrt{2 V_{0}}, \varepsilon \sqrt{2}}\left[2 \sqrt{2 V_{0}} \exp \left(-x_{0}\right)\right], \quad x_{0}>x
\end{array}\right.
$$

Knowing that $F_{1}(X)$ is a solution of the differential Equation (40), then it's easy to check that $X^{\varepsilon \sqrt{2}} F_{1}(X)$ is a solution of the following differential equation:

$$
X^{2} \frac{\mathrm{d}^{2}}{\mathrm{~d} x^{2}} H(X)+X \frac{\mathrm{d}}{\mathrm{d} x} H(X)-\left(2 \varepsilon^{2}-2 X\left(2-\frac{X}{V_{0}}\right)\right) H(X)=0
$$

which is the same as the following differential equation:

$$
\left[-\frac{\mathrm{d}^{2}}{\mathrm{~d} x^{2}}+V(x)+\varepsilon^{2}\right] H(x)=0
$$

for $X=V_{0} \exp (-x)$. Then we conclude that:

$$
H_{1}(x)=\exp \left(\frac{x}{2}\right) W_{\sqrt{2 V_{0}}, \varepsilon \sqrt{2}}\left[2 \sqrt{2 V_{0}} \exp (-x)\right]
$$

and

$$
H_{2}(x)=\exp \left(\frac{x}{2}\right) M_{\sqrt{2 V_{0}}, \varepsilon \sqrt{2}}\left[2 \sqrt{2 V_{0}} \exp (-x)\right]
$$

which are two linearly independent solutions of Equation (49), and since $G\left(x, x_{0}\right)$ is also solution of the differential Equation (13) for $x>x_{0}$, form Equation (47) we can deduce that: 


$$
C_{1}(x)=\lambda_{1} \exp \left(\frac{x}{2}\right) M_{\sqrt{2 V_{0}}, \varepsilon \sqrt{2}}\left[2 \sqrt{2 V_{0}} \exp (-x)\right]
$$

and

$$
C_{2}(x)=\lambda_{2} \exp \left(\frac{x}{2}\right) W_{\sqrt{2 V_{0}}, \varepsilon \sqrt{2}}\left[2 \sqrt{2 V_{0}} \exp (-x)\right]
$$

Finally we get that the Green's function for Morse potential takes the form:

$$
G\left(x, x_{0}\right)= \begin{cases}\left(\lambda_{1}+\lambda_{2}\right) \exp \left(\frac{x+x_{0}}{2}\right) M_{\sqrt{2 V_{0}}, \varepsilon \sqrt{2}}\left[2 \sqrt{2 V_{0}} \exp (-x)\right] W_{\sqrt{2 V_{0}}, \varepsilon \sqrt{2}}\left[2 \sqrt{2 V_{0}} \exp \left(-x_{0}\right)\right], \quad x>x_{0} \\ \left(\lambda_{1}+\lambda_{2}\right) \exp \left(\frac{x+x_{0}}{2}\right) M_{\sqrt{2 V_{0}}, \varepsilon \sqrt{2}}\left[2 \sqrt{2 V_{0}} \exp \left(-x_{0}\right)\right] W_{\sqrt{2 V_{0}}, \varepsilon \sqrt{2}}\left[2 \sqrt{2 V_{0}} \exp (-x)\right], \quad x_{0}>x\end{cases}
$$

and since $G\left(x, x_{0}\right)$ is also a solution of the differential Equation (49) for $x>x_{0}$ or $x_{0}>x$ then the Green's function takes the form:

$$
\begin{aligned}
G\left(x, x_{0}\right)= & \frac{\Gamma\left(\frac{1}{2}+\sqrt{-2 E}-\sqrt{2 V_{0}}\right)}{2 \sqrt{2 V_{0}} \Gamma(1+2 \sqrt{-2 E})} \exp \left(\frac{x+x_{0}}{2}\right) \\
& \left\{\Theta\left(x-x_{0}\right) \exp \left(\frac{x+x_{0}}{2}\right) M_{\sqrt{2 V_{0}}, \varepsilon \sqrt{2}}\left[2 \sqrt{2 V_{0}} \exp (-x)\right] W_{\sqrt{2 V_{0}}, \varepsilon \sqrt{2}}\left[2 \sqrt{2 V_{0}} \exp \left(-x_{0}\right)\right]\right. \\
+ & \left.\Theta\left(x_{0}-x\right) \exp \left(\frac{x+x_{0}}{2}\right) M_{\sqrt{2 V_{0}}, \varepsilon \sqrt{2}}\left[2 \sqrt{2 V_{0}} \exp \left(-x_{0}\right)\right] W_{\sqrt{2 V_{0}}, \varepsilon \sqrt{2}}\left[2 \sqrt{2 V_{0}} \exp (-x)\right]\right\}
\end{aligned}
$$

where $\Theta$ denotes Heaviside's unit step function. A result was found earlier by different methods [11]-[13].

\section{Conclusion}

In this work, we have calculated the Green's function for the Morse potential using the perturbation method in the path integral formalism. This contribution concerns, for the first time, the calculation of the energy Green's function of the system by summing exactly the perturbation series with the introduction of the Fourier transform and some results concerning the Green's function of the ordinary differential equations of the second ordre. We will consider a generalization of this method specialy for other special potentials in the exponential form.

\section{References}

[1] Feynman, R.P. and Hibbs, A.R. (1965) Quantum Mechanics and Path-Integral. McGraw-Hill, New York.

[2] Goovaerts, M.J., Babcenco, A. and Devreese, J.T. (1973) Journal of Mathematical Physics, 14, 554. http://dx.doi.org/10.1063/1.1666355

[3] Lawande, S.V. and Bhagwat, K.V. (1988) Physics Letters A, 131, 8-10. http://dx.doi.org/10.1016/0375-9601(88)90622-6

[4] Grosche, C. (1990) Journal of Physics A: Mathematical and General, 23, 5205-5234. http://dx.doi.org/10.1088/0305-4470/23/22/013

[5] Bhagwat, K.V. and Lawande, S.V. (1989) Physics Letters A, 135, 417. http://dx.doi.org/10.1016/0375-9601(89)90038-8

[6] Lin, D.H. (1997) Journal of Physics A: Mathematical and General, 30, 4365. http://dx.doi.org/10.1088/0305-4470/30/12/022

[7] Lin, D.H. (1998) Journal of Physics A: Mathematical and General, 31, 7577. http://dx.doi.org/10.1088/0305-4470/31/37/015

[8] Bhagwat, K.V. and Lawande, S.V. (1989) Physics Letters A, 141, 321. http://dx.doi.org/10.1016/0375-9601(89)90057-1

[9] Acila, M., Benali, B. and Meftah, M.T. (2006) Journal of Physics A: Mathematical and General, 39, 1357-1366. 
http://dx.doi.org/10.1088/0305-4470/39/6/009

[10] Landau, L. and Lifchitz, E. (1974) Quantum Mechanics. Vol. III, Edition Mir, Moscou.

[11] Khandekar, D.C. and Lawande, S.V. (1986) Physics Reports (Review Section of Physics Letters), 137, 115-229. http://dx.doi.org/10.1016/0370-1573(86)90029-3

[12] Duru, I.H. (1983) Physical Review D, 28, 2689-2692. http://dx.doi.org/10.1103/PhysRevD.28.2689

[13] Fischer, W., Leschke, H. and Muller, P. (1992) Journal of Physics A: Mathematical and General, 25, 3835-3853. http://dx.doi.org/10.1088/0305-4470/25/13/029

[14] Soylu, A., Bayrak, O. and Boztosun, I. (2012) Central European Journal of Physics, 10, 953-959. http://dx.doi.org/10.2478/s11534-012-0018-y

[15] Barakat, T., Abodayeh, K. and Al-dossary, O.M. (2006) Czechoslovak Journal of Physics, 56, 583-590. http://dx.doi.org/10.1007/s10582-006-0122-6

[16] Addis, B. and Schachinger, W. (2010) Computational Optimization and Applications, 47, 129-131. http://dx.doi.org/10.1007/s10589-008-9205-6

[17] Popov, V.N. (2013) High Temperature, 51, 66-71. http://dx.doi.org/10.1134/S0018151X13010124

[18] Földi, P., Benedict, M.G. and Czéryak, A. (2004) Acta Physica Hungarica Series B, Quantum Electronics, 20, 25-28.

[19] Boudjedaa, B., Meftah, M.T. and Chetouani, L. (2007) Turkish Journal of Physics, 31, 197-203. 